\title{
Gradhiva
}

GRADHI

Revue d'anthropologie et d'histoire des arts

$2 \mid 2005$

Autour de Lucien Sebag

\section{Par Simon Njami}

\section{Simon Njami}

\section{OpenEdition}

Journals

Édition électronique

URL : http://journals.openedition.org/gradhiva/523

DOI : 10.4000/gradhiva.523

ISSN : 1760-849X

\section{Éditeur}

Musée du quai Branly Jacques Chirac

\section{Édition imprimée}

Date de publication : 1 novembre 2005

Pagination : 144-145

ISBN : 2-915-133-10-7

ISSN : 0764-8928

\section{Référence électronique}

Simon Njami, «Par Simon Njami », Gradhiva [En ligne], 2| 2005, mis en ligne le 10 décembre 2008, consulté le 15 septembre 2020. URL : http://journals.openedition.org/gradhiva/523

Ce document a été généré automatiquement le 15 septembre 2020.

(C) musée du quai Branly 


\section{Par Simon Njami}

\section{Simon Njami}

1 J'ai lu avec attention le compte rendu que Maureen Murphy a rédigé à propos de l'exposition Africa Remix, et je suis assez perplexe quant à la manière quelque peu manichéenne dont elle a perçu le projet proposé. Commençons par balayer un fait que je ne lui contesterai pas : l'exposition manquait d'espace. Nous avions le choix entre éliminer des œuvres dont le rôle dans la lecture de l'exposition me semblait essentiel et mal les présenter. Je présente donc ici mes excuses aux artistes dont les œuvres n'ont eu droit qu'à un couloir. Puisque nous sommes dans l'interprétation des œuvres et leur lecture, je conclurai ce chapitre sur les remarques de Maureen Murphy à propos de l'œuvre de Mohamed El Baz qu'elle lit comme une critique de l'exposition alors que, pour avoir assisté à cet ajout de dernière minute, je sais que cet artiste faisait uniquement allusion au sponsor principal de l'étape parisienne, Total, et à la présence au vernissage du président en exercice de l'Union Africaine qui s'appelait encore, il y a peu, OUA. Il est donc bon, parfois, de prendre quelques renseignements avant de tirer des conclusions hâtives et idéologiquement conditionnées. Comme est idéologiquement conditionné le fait que l'auteur, dans sa hâte d'exposer ses thèses, ait pu écrire à propos des meubles de Cheick Diallo : « un des seules réalisations inédites de cette exposition ». Pour son information, je lui rappellerai donc que le centre Georges-Pompidou constitue l'étape d'une tournée. Les œuvres créées pour Africa Remix sont celle de Lara Baladi, Bili Bidjocka, Pascale Marthine Tayou, Pume, Antonio Ole, Mohamed El Baz, Mechack Gaba, Abdoulaye Konaté, Andries Botha, Akinbode Akinbiyi, Joel Andrianomeisora, Balthazar Faye et Patrice Félix Tchikaya. Peut-être en ai-je oublié au passage. Mais qu'importe. Il me semble que ce « détail » parmi d'autres éclaire sur le ton général de l'article dont je me propose de reprendre les principaux points.

2 Il n'y a rien de l'esprit pionnier des aventuriers du xIX ${ }^{e}$ siècle, bien au contraire. Cette exposition est une somme, l'étape d'une réflexion que j'ai entamée il y a plus de quinze ans sur le sens de la contemporanéité en Afrique. Une contemporanéité qui ne serait pas définie selon les critères occidentaux, mais selon des critères des Africains euxmêmes. L'Afrique, n'en déplaise à l'auteur du compte rendu, n'est pas pour moi un mystère. Je crois la connaitre de l'intérieur comme de l'extérieur et avoir passé plus de temps avec des artistes africains qu'elle n'en aura jamais l'occasion. Partir d'une vision 
ethnocentrique qui voudrait que toute analyse soit nécessairement issue de l'Europe fait partie des quelques lieux communs qu'Africa Remix entendait remettre en question. Si Maureen Murphy ne s'imaginait pas que la terre, le corps ou l'esprit sont le propre des Africains et véhiculent nécessairement une vision anthropologique, elle se serait aperçue que, précisément, ces thématiques pourraient s'appliquer à n'importe quel art. L'exposition s'est concentrée sur les réponses plastiques qu'apportaient ces artistes à des questions universelles. Quant à comparer Africa Remix avec les Magiciens de la terre sur l'analyse de laquelle je suis d'accord, c'est, à mon sens, faire preuve de la plus achevée mauvaise foi. Je maintiens au passage être l'auteur du concept de cette exposition. Que Jean-Hubert Martin ait pensé à accueillir une " exposition africaine " à un moment ou un autre n'a que peu d'importance en l'occurrence. C'est tout à fait légitime de sa part, son intérêt pour ce continent n'étant plus à démontrer. D'autres, sans doute, ont eu cette idée et, quant à moi, j'ai été le commissaire de bien des expositions d'art contemporain à travers le monde. Mais voilà que soudain l'exception française se met à l'œuvre et que des questions qui n'ont pas lieu d'être à Düsseldorf comme à Londres deviennent ici matière à faux débat. Je ne comprends d'ailleurs pas, dans son exposé, comment l'auteur explique et justifie la comparaison avec les Magiciens. Il est tellement plus simple d'affirmer que de démontrer.

Qu'un Africain, pour des raisons historiques, soit né à Moscou, comme c'est le cas de l'Algérienne Zoulikha Bouabdellah semble un problème. Or, si cela constitue un problème, c'est bien que l'exposition aura au moins atteint l'un de ses objectifs. Le fait qu'une jeune Algérienne née à Moscou côtoie un Ivoirien âgé vivant en Côte d'Ivoire dans une même exposition ne postule pas de l'idée "d'une essence de l'art africain ", bien au contraire. J'ai tenu à montrer la diversité des pratiques et des individualités qui formaient aujourd'hui ce que l'on appelle l'art africain contemporain. Il s'agissait de démontrer par les faits que diverses tendances traversent cette création, parce que cette création est nourrie par diverses expériences. Les artistes comme Bruly Bouabré n'ont pas d'âge. Ils participent de la même logique créatrice que des Paulo Capela, Georges Adeagbo ou Pume, qui sont pourtant de générations différentes. Se poser des questions sur l'âge, c'est refuser de voir et, partant, s'interdire de comprendre.

4 Demeurent deux points encore, qui confirment hélas le contresens total de cette critique. Le premier est celui de la négritude. J'ai effectivement cité dans mon texte Senghor et Sartre, parmi d'autres auteurs ou penseurs d'ailleurs, mais qu'importe. Comprendre une citation de Senghor à l'aune de sa "négritude ", c'est ne rien comprendre du tout. C'est oublier qu'il fut également le chantre de l'universalité. C'est oublier qu'en rassemblant l'Afrique en un seul lieu, il n'est plus question de couleurs de peau ou de races, mais d'un questionnement sur l'histoire complexe de ce continent et de son avenir. C'est oublier la présence de Jane Alexander ou de Mounir Fatmi. Quant à la référence à Wole Soyinka sur la tigritude du tigre, c'est oublier que le prix Nobel est revenu lui-même sur ce bon mot il y a des années et l'a confirmé lors du colloque alors que je le taquinais à ce propos.

5 Quant à l'apologie de la pauvreté dont je serais devenu l'un des chantres, s'il est une critique à laquelle je croyais pouvoir échapper, c'est bien celle-là. Que l'on ne soit pas capable de comprendre un texte est une chose admissible. Mais que l'on soit aveugle au point de prétendre que l'exposition " traduit une fascination pour la friche » est simplement incompréhensible à mes yeux. De toute évidence, Maureen Murphy n'a pas 
vu la même exposition que de nombreuses autres personnes. Et celle qu'elle a vue, il me semble ne l'avoir jamais pensée. 